\title{
Tracking performance as a function of distorted knowledge of results'
}

\author{
Frank J. Vattano \\ UNIVERSITY OF DENVER
}

\begin{abstract}
Abstraet
The influence of distorted knowledge of results on tracking performance of 32 male and 32 female Ss was examined. Results indicate the performance of the male Ss was uninfluenced by the various distortion conditions. For the female Ss distorted knowledge of results had a significant effect upon tracking performance, with the condition of least distortion resulting in poorer performance, than under the condition of the greater distortion.

\section{Problem}

Several studies in the literature indicate that under conditions of no specific knowledge of results, performance in perceptual motor tasks improves as a simple function of number of trials (Ammons, 1954; Andreas, 1960). Annet \& Kay (1957) have stated that knowledge of results may be of two different kinds, some of which may be extrinsic (an end score provided by $\mathrm{E}$ or some type of feedback circuit) and some intrinsic to the task (like proprioceptive and visual feedback). That a task may possess what might be called intrinsic knowledge is evidenced by the studies cited in Ammons (1954) and also by Andreas (1960), where no significant differences in performance were obtained in various types of perceptual motor tasks between Ss who had received specific knowledge both during and at the end of each trial, and those who received no knowledge of results. In an attempt to study whether Ss rely upon extrinsic or intrinsic knowledge of results, the present study employed distorted extrinsic knowledge and accurate intrinsic knowledge. It was hypothesized that with intrinsic knowledge available, extrinsic distorted knowledge of results would have no significant effect upon tracking performance. Method

The Ss were 64 undergraduate psychology students, 32 male and 32 female with no previous tracking experience. The apparatus used was a simplified electronic tracking apparatus (SETA) designed by Gain and Fitts (1958). The apparatus was housed in two cabinets. The S's component contained a power supply, tracking display, and control knob. S's task was to keep a pointer needle at the null point by manipulating his control knob in a compensatory tracking arrangement. The task was described to the Ss as a simulated missile tracking task. The E's cabinet contained an analog computer element, problem generator, programer, scoring display, and operating controls. The S's score was indicated by a voltmeter with a scale divided into 100 units located in the center of the front panel of the E's cabinet. The score given to $\mathrm{S}$ was indicated by another voltmeter with a scale divided into 200 units, located to the S's left. The E's unit was modified to enable $\mathrm{E}$ to distort the S's score in a plus or minus fashion from 0 to $30 \%$, by means of a variable resistor. This score appeared on the S's meter and the S's 'true" score appeared on the E's voltmeter.

Eight males and eight females were assigned to each of four conditions: Group I, true knowledge; Group II, plus and minus 5\% and $10 \%$ distortion; Group III, plus and minus $15 \%$ and $30 \%$ distortion; and Group IV, no knowledge. Each S was given $201-$ min. trials with a 30-min. intertrial interval. Of the 20 trials, the distorted knowledge groups (II and III) received four trials of "true" knowledge and four trials of plus and minus the two distortion conditions, assigned in a prearranged order. The Ss were isolated from the $\mathrm{E}$ and were instructed to keep the pointer on the zero mark by manipulating the control knob. They were instructed to read their score to the $\mathrm{E}$ after each trial (except for Group IV) which represented how far off target (0 to 200 miles) they were on any one trial. This reading constituted the extrinsic knowledge. Ss continuous tracking feedback (visual and kinesthetic) constituted the intrinsic knowledge.

\section{Results}

The results in terms of time on target in mean integrated absolute error for the last five acquisition trials are presented in Table 1. An analysis of variance over the different knowledge conditions indicated a significant effect $(F=5.42 ;$ df $=3,63 ; P<.01)$. Sex differences were also highly significant $(F=21.80$; $\mathrm{df}=1,63$; $\mathbf{P}<.001)$. Separate analysis of males indicated no sig-

Table 1. Analysis of Mean Scores for Last Five Tracking Trials in Percent

Time On Target

\begin{tabular}{lccccccc} 
Comparison & N & \multicolumn{5}{c}{ Groups } & F \\
& & I & II & III & IV & \\
& & & & & & \\
All Conditions & 64 & 61 & 84 & 68 & 75 & $5.42^{* *}$ \\
Male Ss & 32 & 55 & 72 & 62 & 60 & 1.88 NS \\
& & & & & & \\
Female Ss & 32 & 67 & 96 & 74 & 91 & $4.25 *$ \\
& & & & & & \\
Male vs. Female & 64 & & & & & $21.80 * *$ \\
$*$ & & & & & & \\
$* *$ & $=\mathrm{P}<.05$ & & & & &
\end{tabular}


nificant differences in tracking performance $(F=1.88$; $\mathrm{df}=3,31$ ). However, analysis of female tracking performance yielded a significant difference $(F=4.25$; $\mathrm{df}=1,63 ; \mathrm{P}<.05)$. The differences between Groups I and II $(\mathrm{t}=28.33 ; \mathrm{P}<.01)$, Groups I and IV $(\mathrm{t}=23.73$; $\mathrm{P}<.05)$ and Groups II and III $(\mathrm{t}=22.00 ; \mathrm{P}<.05)$ were significant. Other comparisons between individual groups yielded non-significant differences.

Considering all Ss together the hypothesis was not confirmed. When considering male and female Ss separately however, the hypothesis was confirmed for the males but not for the females. Reference to Table 1 shows that for both males and females, condition II (the least distorted knowledge condition) resulted in the poorest performance.

\section{Diseussion}

The results provide partial evidence for the hypothesis that knowledge of results provided by visual and kinesthetic means (intrinsic) operates independently of knowledge of results provided by an external source (extrinsic). With female Ss however, this hypothesis was not supported. Also there arises the question of why the greater amount of distorted knowledge resulted in superior performance over the lesser amount of distortion.

Perhaps the reason for sex differences in performance is related to the nature of the task. Women, generally speaking, do not (and are not expected) to perform as well on tasks related to motor performance in a quasi military setting. Albeit the study was not designed to bring out cultural factors in performance, it is not unlikely that men in general have had more previous experience in tasks similar to this, whereby transfer of this previous experience might have facilitated their performance. Perhaps the field dependence-independence notion of Witkin (1959) is also applicable to this study.

No information was obtained from the experiment which would allow one to make any conclusions regarding the discrepancy between the amount of distortion and performance. Even though Ss expressed no sign of distrust upon receiving distorted scores, it is quite possible that these large distortions of up to $30 \%$ of actual scores were not taken on their face value. On the other hand, it is possible that distortions of a lesser amount were more compatible with S's own estimate of performance.

\section{Referenees}

AMMONS, R. B. Knowledge of performance. WADC Tech. Rep. , 54-14, 1954.

ANDREAS, B. G. Experimental psychology. New York: Wiley, 1960. P. 429.

ANNETT, J., \& KAY, H. Knowledge of results and "skilled performance." Occup. Psychol., 1957, 31, 69-79.

GAIN, P., \& FITTS, P. M. A simplified electronic tracking apparatus (SETA). WADC Tech. Rep., 58. Laboratory of Aviation Psychology, The Ohio State University.

WITKIN, H. A. The perception of the upright.Scient. Amer., 1959, 200, 50-56.

\section{Note}

1. This work is based on a thesis submitted to the Graduate School of The Ohio State University in partial fulfillment of the requirements for the M. A. degree in psychology. 